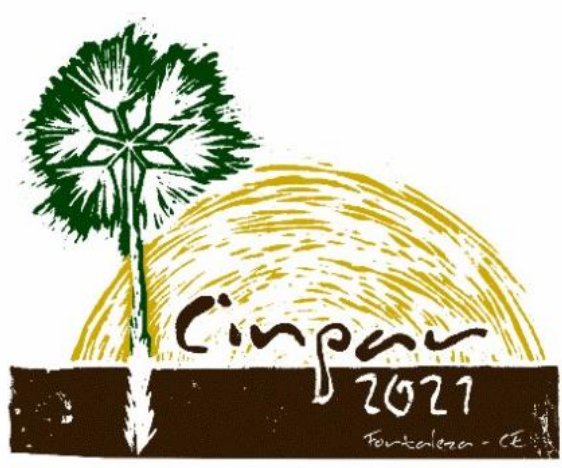

XVII Congresso Internacional sobre Patologia e Reabilitação das Construções

XVII Congreso Internacional sobre Patología y Rehabilitación de las Construcciones

XVII International Conference on Pathology and Constructions Rehabilitation

FORTALEZA (Brasil), 3 a 5 de junho de 2021

https://doi.org/10.4322/CINPAR.2021.155

\title{
Avaliação de Desempenho por meio de Ensaio Morfológico aplicado a arquitetura hospitalar com foco na avaliação técnica
}

\section{Performance Evaluation through Morphological Test applied to hospital architecture with a focus on technical evaluation}

\author{
Alyria DONEGÁ ${ }^{1}$, João PANTOJA \\ ${ }^{1}$ Universidade de Brasília, Brasília, Brasil, alyria.donega@gmail.com \\ ${ }^{2}$ Universidade de Brasília, Brasília, Brasil, joaocpantoja@gmail.com
}

\begin{abstract}
Resumo: Este artigo tem por finalidade apresentar uma avaliação qualitativa por meio de avaliação de desempenho com base no ensaio das dimensões morfológicas desenvolvido na Universidade de Brasília UNB, adaptada pelos autores visando a aplicação do método à arquitetura hospitalar. O método adaptado vem sendo desenvolvido visando o gerenciamento de edificações existentes quanto a sua manutenção, confiabilidade, extensão de vida útil; além da preservação de patrimônio histórico da edificação citada. A avaliação consiste num estudo técnico dos elementos que compõem a edificação com base nos projetos existentes, levantamentos fotográficos e levantamentos in loco. 0 estudo pretende focar na verificação dos pontos críticos que necessitam de manutenção, assim como a conformidade quanto as normas do Ministério da Saúde, de acessibilidade, saídas de emergências, manutenção e outras correlatas. Os resultados identificaram, principalmente, a necessidade de adequação da quanto as normas de prevenção a incêndios e acessibilidade vigentes.
\end{abstract}

Palavras-chave: avaliação de desempenho; inspeção predial; arquitetura hospitalar; performance evaluation; building inspection; hospital architecture;

\section{Introdução}

O hospital consiste em uma instituição complexa por integrar, ou dever considerar, em seu planejamento (pré ou pós-ocupação) fatores das mais diversas áreas da arquitetura como estrutura, sistema construtivo, revestimentos, instalações, equipamentos, tecnologia, manutenção e gestão. Elementos estes que influenciarão diretamente na qualidade da edificação e a eficiência dos serviços de saúde prestados, elementos estes pensados na concepão inicial da edificação ou numa futura consideração pós-ocupação.

Nesse contexto da Avaliação pós-ocupação no Brasil, segundo Ornstein e Del Cario (2017), pode-se observar o início da pesquisa em meados da década de 60 onde o Prof. Dr. Ualfrido del Carlo dedicou-se a temática correlacionada em seu doutorado na Faculdade de Arquitetura e Urbanismo da Universidade de São Paulo (FAU/USP) e trabalhos posteriores, perpassando pela avaliação do desempenho ambiental até a fase avaliação pós-ocupação, na qual nos encontramos. Pesquisas estas que visavam avaliar o sistema construtivo (ONO et al., 2013), incluindo sistemas construtivos inovadores (ONO et al., 2017; ORNSTEIN; ONO; OLIVEIRA, 2017); habitações sociais (ORNSTEIN, 1990), o desempenho do edifício (ORNSTEIN, 1997; ORNSTEIN et al., 2009; ORNSTEIN; LEITE; ANDRADE, 1999), a eficiência e a otimização dos recursos (GUELLI, 2010; ROMÉRO, 2016). 
Ornstein (2017) esclarece ainda que a avaliação pós-ocupação vem sendo desenvolvida e aplicada, em sua maioria, em estudos relacionados as habitações coletivas, e em menor expressão nas demais tipologias da arquitetura. Sendo que, conforme exposto pela mesma autora (ORNSTEIN et al., 2009), em estabelecimento de saúde, os itens a serem avaliados diferem daqueles aplicados a habitações, necessitando de uma avaliação mais técnica do que baseada na opinião do usuário, sendo necessário, portanto, adequações e pesquisas complementares direcionadas. Como o caso dos estudos aplicados pela FIOCRUZ no Rio de Janeiro, aplicada por uma equipe multidisciplinar composta por Castro, Lacerda e Penna (CASTRO; LACERDA; PENNA, 2004; PENNA et al., 2002). Dentro desse aspecto, há de se pensar num método de avaliação de desempenho voltado a arquitetura hospitalar. Vale destacar-se que segundo Ornstein (1992) não é cabível a avaliação de desempenho sem considerar a opinião do usuário ou da considerações sobre este no usufruto da edificação, apesar de se ter como foco a avaliação mais técnica para este tipo de edificação.

Uma vez que quando a edificação hospitalar deixa de ser um equipamento de qualidade e apresenta baixa eficiência ou necessidade de adequação, há de se pensar na reabilitação da mesma. Podendo ser relacionada a correção de patologias e da degradação da edificação (HELENE, 1992; THOMAZ, 1989); a melhoria do desempenho térmico e energético (ROMERO; SALES, 2016), a considerações do desempenho econômicofinanceiro (MASCARÓ, 1985; OLIVEIRA, 2016), ao desempenho dos serviços prestados por meio do aprimoramento do planejamento arquitetônico (GÓES, 2011; KARMAN, 1994), a otimização dos processos envolvidos (ALÁSTICO; TOLEDO, 2013), a logística e sistematização (BROSS, JC; 1989 apud GUELLI; ZUCCHI, 2005), maximizar a segurança e conforto dos usuários, ou ainda "a redução dos impactos ambientais por meio do aumento da eficiência dos sistemas componentes do espaço construído" (ROMERO; TEIXEIRA, 2016); utilizando, sempre que possível, energias renováveis. Podendo considerar ainda, a otimização e eficiência em relação ao funcionamento, a redução do impacto ambiental do meio em que se insere, a produtividade, o mínimo desperdício e a qualidade; além de relacionar ao preceito de preditividade, atividade voltada ao prévio estudo de impactos sobre a edificação (KARMAN, 1994).

Outro fator que colabora com a visualização da necessidade do desenvolvimento de um método de avaliação do ambiente construído aplicado a arquitetura hospitalar, com vistas a sua manutenção e reabilitação, é a recorrente esclarecimento quanto ao alto custo de investimento para a construção de novos hospitais, onde $60 \%$ do custo corresponde as obras e $40 \%$ aos equipamentos (GÓES, 2011; HELENE, 1992; KARMAN, 1994; ORNSTEIN, 1992; THOMAZ, 1989). Onde até mesmo a terceirização de alguns serviços hospitalares como a lavanderia industrial, centrais de esterilização e nutrição, geram uma redução da área do edifício e influenciam diretamente no custo de construção/manutenção. Além do fato da constante evolução da medicina e tecnologia - e consequentemente de seus equipamentos e ambientes, que de certa forma exigem da edificação hospitalar o constante desenvolvimento e necessidade de adequação, de forma a acompanhar esta evolução, pois nem sempre a edificação é compatível com as necessidades daquele momento. Devendo visar, sempre que possível, a redução de custos e tempo e o aumento da qualidade e eficiência da edificação.

Eficiência esta que pode ainda ser extrapolada e relacionada a sustentabilidade e a redução do impacto ambiental de uma edificação, de grande impacto como é o caso de hospitais. Sendo esta temática discutida recorrentemente por pesquisadores da área, onde se destacam, principalmente, o desperdício de recursos, o impacto ambiental e o alto consumo de água e energia pelas edificações (KARMAN, 1994; ROMERO; SALES, 2016). Ações estas que podem ser vantajosas não somente na visão de desenvolvimento sustentável, mas também por influenciar econômico-financeiramente a edificação ao longo dos anos.

Desta forma, há de se deduzir que o aumento do desempenho e eficiência do ambiente construído seria vantajoso em relação a construção de um complexo hospitalar totalmente novo, devido a aspectos tanto econômico-financeiro como de impacto ambiental. Neste contexto que se insere, portanto, a necessidade de uma avaliação do ambiente construído e ocupado por meio de inspeções periódicas, de modo a verificar se o mesmo desempenha a função a que se destina corretamente, ou ainda se há maneiras de torna-lo mais eficiente. Considerando que as inspeções periódicas devem atuar nas seguintes verificações: inexistência de 
danos, pequenos danos, danos importantes, danos emergenciais e alarme, segundo Souza e Ripper (1998). Sendo caracterizados, respectivamente por necessidade de: nenhuma atitude, correção por pessoal não especializado, correção por empresas de pequeno ou médio porte com responsável técnico, convocação de especialistas para providências e de escoramento parcial ou total - passível de ruína. Pois segundo os autores, as manutenções e inspeções, deverão verificar principalmente os locais passíveis de potencial deterioração e desgaste.

Portanto, aqui se evidencia a necessidade de um método de avaliação periódico das edificações, com vistas a verificação não só da eficiência da edificação, mas também da verificação de patologias. Sendo que no aspecto da inspeção periódica, há de se estabelecer uma análise preliminar de forma a indicar os pontos nos quais se verifique a necessidade de estudos mais aprofundados das problemáticas encontradas. Sugerindo a aplicação de um método voltado a avaliação técnica da edificação e seus sistemas com uma sistemática de representação para os pontos de gravidade e/ou urgência. Por fim, nesse estudo foi aplicado o método de avaliação de desempenho das dimensões morfológicas ampliado com um método qualitativo para determinação de gravidade/urgência aplicado ao Hospital Sarah Centro para verificação da conformidade do método quanto ao objeto de estudo.

\section{Método}

As dimensões morfológicas têm sido desenvolvidas ao longo dos anos - tabela 1 , sendo resultado do grupo de pesquisa de professores da Faculdade de Arquitetura e Urbanismo da Universidade de Brasília - FAU/Unb, denominado Dimensões Morfológicas do Processo de Urbanização - DIMPU, de 1984. Que além da possibilidade de avaliação do espaço urbano, método de avaliação mais usual das dimensões, pode ser aplicado a avaliação do espaço arquitetônico, seja pré ou pós-execução, da escala macro a mico - urbano ao edifício, conforme esclarece Kohlsdorf e Kohlsdorf (2017) e Holanda (2010). Contudo, para fins metodológicos, será utilizada a categorização exposta pelos autores em 2017 - conforme tabela 1, que abrange, direta ou indiretamente, as outras duas dimensões de Holanda (2010). A título de ambientação, na figura 1 há uma breve consideração do que se trataria cada dimensão. Sendo válido destacar que, conforme os mesmos autores, as dimensões ainda estão inseridas no contexto de três macrodimensões: éticas, estéticas e ecológicas que permeiam todo o contexto de desempenho das edificações e que devem ser consideradas no momento de suas aplicações.

Quadro 1 - Resumo da evolução das dimensões morfológicas na arquitetura. Fonte: Tenório (2012 p. 30) adaptado pela autora.

\begin{tabular}{|l|l|l|l|l|l|}
\hline \multicolumn{1}{|c|}{ 4 funções } & 7 Dimensões & 7 Dimensões & $\mathbf{6}$ Dimensões & $\mathbf{8}$ Dimensões & 6 Dimensões \\
\hline $\begin{array}{l}\text { Hiller } \\
\text { Leaman, } \\
1974\end{array}$ & $\begin{array}{l}\text { Holanda e } \\
\text { Kohlsdorf, G; } \\
1994\end{array}$ & $\begin{array}{l}\text { Kohlsdorf, } \\
\text { M.E.; } \\
1996\end{array}$ & $\begin{array}{l}\text { Kohlsdorf, } \\
\text { M.E.; } \\
2010\end{array}$ & $\begin{array}{l}\text { Holanda, } \\
2010\end{array}$ & $\begin{array}{l}\text { Kohlsdorf, M.E } \\
\text { e Kohlsdorf, G. } \\
2017\end{array}$ \\
\hline $\begin{array}{l}\text { continente de } \\
\text { atividades }\end{array}$ & funcional & Funcional & Funcional & Funcional & Funcional \\
\hline & de copresença & de copresença & de copresença & Sociológica & Copresencial \\
\hline $\begin{array}{l}\text { modificação } \\
\text { climática }\end{array}$ & bioclimática & Bioclimática & Bioclimática & Bioclimática & Bioclimática \\
\hline $\begin{array}{l}\text { modificação de } \\
\text { recursos }\end{array}$ & econômica & Econômica & $\begin{array}{l}\text { econômica- } \\
\text { financeira }\end{array}$ & Econômica & $\begin{array}{l}\text { econômica- } \\
\text { financeira }\end{array}$ \\
\hline & topoceptiva & Topoceptiva & Topoceptiva & Topoceptiva & Topoceptiva \\
\hline $\begin{array}{l}\text { expressão } \\
\text { simbólica }\end{array}$ & simbólica & Expressiva & $\begin{array}{l}\text { expressivo- } \\
\text { simbólica }\end{array}$ & Simbólica & $\begin{array}{l}\text { expressivo- } \\
\text { simbólica }\end{array}$ \\
\hline & emocional & Afetiva & & Afetiva & \\
\hline & & \multicolumn{5}{|l}{} & Estética & \\
\hline
\end{tabular}




\begin{tabular}{|c|c|c|c|}
\hline Dimensão & Conceito simplificado & Esfera & $\begin{array}{c}\text { Caráter } \\
\text { (HOLANDA, 2010) }\end{array}$ \\
\hline funcional & $\begin{array}{l}\text { Atende as exigências e } \\
\text { necessidades? }\end{array}$ & Física & Instrumental \\
\hline copresencial & $\begin{array}{l}\text { possibilidade de encontros } \\
\text { casuais e reclusão }\end{array}$ & Social & Instrumental \\
\hline bioclimática & $\begin{array}{l}\text { expectativa de conforto } \\
\text { metabólico dos indivíduos } \\
\text { com o meio que se encontram }\end{array}$ & Física & Instrumental \\
\hline $\begin{array}{l}\text { econômica- } \\
\text { financeira }\end{array}$ & custos $\mathrm{x}$ benefícios & Pragmática & Instrumental \\
\hline topoceptiva & $\begin{array}{l}\text { orientação espacial e } \\
\text { identificação dos lugares }\end{array}$ & Emocional & Expressivo \\
\hline $\begin{array}{c}\text { expressivo- } \\
\text { simbólica }\end{array}$ & $\begin{array}{l}\text { vínculos emocionais x fruição } \\
\text { estética e características dos } \\
\text { lugares }\end{array}$ & Emocional & Expressivo \\
\hline
\end{tabular}

Figura 1 - As seis dimensões de avaliação do desempenho morfológico dos lugares. Fonte: Kohlsdorf e Kohlsdorf (2017 p. 469) adaptado pela autora.

Sendo válido esclarecer que o foco dessa avaliação pode ser pontual quanto generalizada conforme necessidade ou capacidade do momento em que for aplicada. Devendo-se estudar as questões de avaliação pós-ocupação e avaliação de desempenho aplicados a arquitetura hospitalar. Avaliações estas que, por sua vez, devem ser categorizadas de acordo com a complexidade das atividades desempenhadas e suas respectivas funções dentro do contexto da edificação, a fim de realizar uma pesquisa mais objetiva.

Pois conforme os autores, a avaliação de desempenho segundo as dimensões morfológicas tem como objetivo verificar as qualidades de cada lugar, seja urbano ou arquitetônico, segundo as suas particularidades. Assim como há de se deduzir que com a categorização dos fatores a serem avaliados, há de se estabelecer uma avaliação mais sistematizada e direcionada, incluindo para a divisão das atividades pela equipe técnica de avaliação ou mesmo do levantamento da opinião do usuário.

Nesse contexto, segundo Kohlsdorf e Kohlsdorf (2017), com a aplicação das dimensões morfológicas como norteadores da avaliação o estudo tende a considerar todos os aspectos passíveis de influência sobre uma edificação. Dimensões estas passíveis tanto a avaliação de desempenho (mais técnica) quanto a avaliação do ponto de vista do usuário, ou seja, uma avaliação pós-ocupação concreta. Sendo válido destacar que no presente estudo, nesse primeiro momento, pretende-se focar na avaliação técnica.

\section{Estudo de Caso}

O Hospital Sarah Centro - ou Sarah Brasília, consiste em um complexo hospitalar com aproximadamente $68.000 \mathrm{~m}^{2}$ e capacidade de 300 leitos, voltado para o atendimento especializado ao aparelho locomotor e dispõe de toda a estrutura básica de um hospital especializado, abrigando os setores de apoio e atendimento, exceto o atendimento imediato - urgência e emergência, conforme classificação da RDC 050 (ANVISA, 2002). Sendo escolhido devido a sua relevância no campo da arquitetura, método construtivo, e eficiência no atendimento.

O Hospital foi planejado em busca otimização do espaço, alta produtividade e eficiência, alta tecnologia, ambientes humanizados e a aplicação de métodos naturais para o fornecimento do conforto ambiental. Implantado por meio de um sistema construtivo industrializado com peças pré-moldadas. O projeto é de 
autoria do arquiteto João Filgueiras Lima, foi inaugurado em 1974 , possui atualmente $67.684,54 \mathrm{~m}^{2}$ construídos e é considerado um dos melhores hospitais do mundo. Suas especialidades de atendimento são ortopedia, pediatria do desenvolvimento, neurologia, neurocirurgia, genética médica, cirurgia reparadora e oncologia e desenvolve programas de reabilitação neurológica, neurorreabilitação em lesão medular e auxílio ao diagnóstico e tratamento.

O Sarah Brasília está localizado no Setor Médico Hospitalar Sul (SMHS), região central do Plano Piloto (RA-I) de Brasília. Em um terreno com aproximadamente $58.000,00 \mathrm{~m}^{2}$, projetado com volumes compactos a fim de garantir áreas externas livres e espaço para futuras ampliações, como o auditório e o Sarinha (LIMA, 2012). Localizado entre três vias coletoras - em uma zona predominantemente comercial com mais de seis pavimentos, com a presença de pouca vegetação, com a presença de várias edificações e locado ao lado do HBDF.

O Sarah Brasília é composto por dez volumes, que compreendem o estacionamento enterrado, o Sarinha bloco A à D, a Escolinha - que foi desativada, o Complexo Principal, o Auditório e o Edifício Sede. Cada bloco possui funções bens específicas utilizados de forma independente umas das outras. Sendo que a presente avaliação terá como foco o complexo principal.

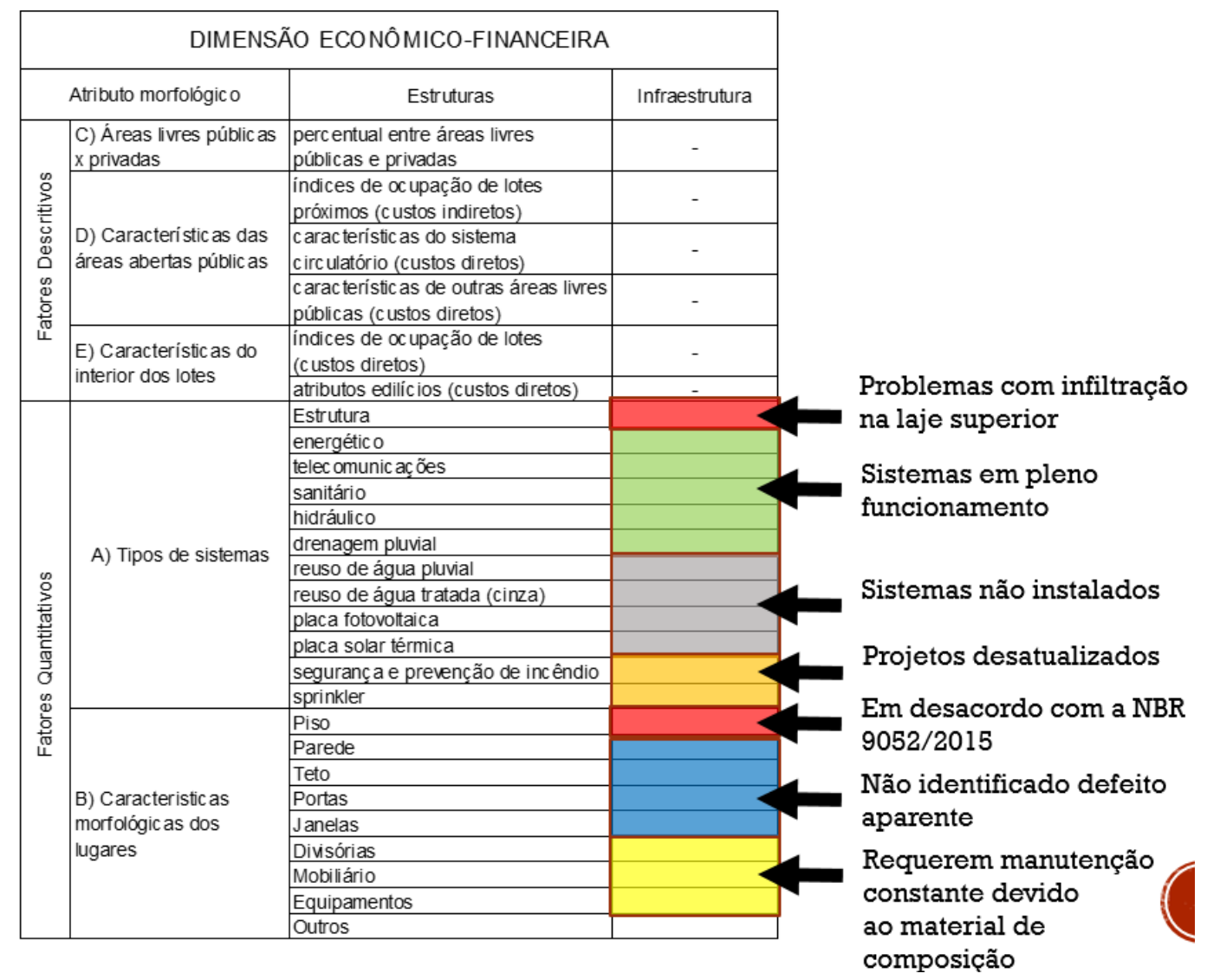

Figura 2 - Estudo qualitativo desenvolvido para definição e consideração do gravidade e urgêncis dos pontos analisados para posterior quantificação

Em praticamente todo o complexo o piso vinílico apresenta emendas com saliências de até um $\mathrm{cm}$, que do ponto de vista da acessibilidade não causa incômodos, porém pode incomodar a transferência de pacientes em macas. Sendo este padronizado em praticamente em todo o complexo, exceto no pátio na entrada do Sarinha - que apresenta danos - e das oficinas localizadas nos blocos D e E, consistindo também nos lugares que apresentam um pequeno acumulo de sujeira. Contudo, esses tipos de pisos estão em desacordo com a RDC 050 (BRASIL, 2002) a qual especifica que os pisos em estabelecimento de saúde não devem conter juntas. Em relação as estruturas, paredes fixas, paredes semi-móveis, acessos visitáveis e móveis, não foram constatadas patologias aparentes. 
Em relação ao acesso ao Sarah Brasília, houve relatos sobre a dificuldade de estacionar nos estacionamentos públicos próximo ao hospital, uma vez que a área comercial do entorno possui grande demanda. No estacionamento de uso exclusivo dos funcionários, por sua vez, as principais problemáticas se relacionam a inadequação da sinalização de emergência e rota de fuga - escada metálica, sem espelhos e sem sinalização visual.

No edifício sede foram identificados a inadequação da sinalização de emergência; subdimensionamento na distribuição dos extintores e/ou tipo inadequado, inadequação do guarda-corpo da biblioteca do 11응 pavimento, necessidade do redimensionamento da Reserva técnica de incêndio (RTI), deficiência na iluminação de emergência, ausência de chuveiros automáticos (exigido para edificações acima de 12 metros de altura para a área) e inadequação da rota de fuga - necessita de correção da ventilação natural das escadas. A passarela que conecta o edifício sede ao complexo principal, apresentava inadequação da escada localizada entre as edificações, quanto as normas de segurança, e o fator de "túnel de vento", uma vez que devido aos materiais construtivos da mesma - policarbonato e aço, a passarela possuía a tendência ao aquecimento e por tal razão instalaram um sistema de ventilação mecanizada demasiado forte para o ambiente. No auditório, foi verificada a presença de infiltrações e consequentes danos ao piso e mobiliário do mesmo em sua entrada, além da inadequação quanto as normas de sinalização e extintores de incêndio

No complexo principal, nos subsolos, foi verificada a inadequação das escadas de rota de fuga, sinalização de emergência, iluminação de emergência, extintores e problemas com alguns chuveiros de sprinkler. Além de falhas na estrutura de proteção aos técnicos da área de raio-x - não era totalmente isolado - e a obstrução do acesso aos equipamentos de segurança, extintores e hidrantes. A rampa que conecta os subsolos ao térreo, também apresentava problemas quanto a inadequação de piso e de corrimão quanto a acessibilidade e segurança. Na torre de internações, no primeiro pavimento, os dormitórios médicos foram convertidos em dormitórios para acompanhantes pelos próprios funcionários, uma vez que se trata do andar de enfermagem pediátrica e os acompanhantes possuem o direito de pernoite junto ao paciente e o hospital não possui esse tipo de estrutura. Nos jardins internos, se verificou que algumas vigas possuíam grande exposição a umidade e água. Assim como a presença de infiltrações e vegetação nas lajes de cobertura do edifício sede e do bloco de internações e suas varandas - nas quais se dificultava o uso e um sentimento de insegurança expressado pelos funcionários quanto a utilização do local. Ambos casos necessitam, portanto, uma maior investigação quanto a presença de patologias e/ou danos as estruturas.

\section{Conclusões}

Em relação a avaliação técnica, nota-se que em praticamente todo o complexo hospitalar há inadequações quanto a sinalização de emergência, iluminação de emergência, dimensionamento de extintores, obstrução de acesso aos equipamentos de segurança, inadequação das escadas de rota de fuga e rampas - incluindo inadequação de guarda-corpo e corrimão - e infiltrações nas coberturas e varandas - exceto sheds. Apresentando, portanto, necessidade de adequação das questões de segurança, acessibilidade e manutenção da edificação como ponto principal, além de um estudo mais aprofundado quanto a questão das infiltrações nas lajes de cobertura do bloco de internações e no edifício sede. Além do estudo da correção dos pisos do complexo hospitalar - quanto a emendas - para adequação a legislação vigente.

Em relação a opinião dos usuários, não houve queixas quanto a limpeza de todo o complexo ou de banheiros, nem do estado de conservação como um todo. Quanto aos ambientes, afirmaram possuir boa iluminação e conforto térmico - exceto os meses mais quentes de Brasília, necessitando portando de um estudo mais aprofundado. Apresentando insatisfação apenas quanto ao estacionamento público fora do complexo hospitalar e a ausência de lanchonete no complexo - para acompanhantes e visitantes.

Em relação a avaliação qualitativa, com base nos dados levantados, não foram verificados pontos problemáticos apresentados além do exposto pela opinião dos usuários e da avaliação técnica. $O$ que expõe que as recomendações de manutenção quanto a questões de segurança do Sarah Brasília se encontra em um

Avaliação de Desempenho por meio de Ensaio Morfológico aplicado a arquitetura hospitalar 
aspecto mais técnico, uma vez que o projeto do mesmo não é revisto a aproximadamente 40 anos e as normas aplicáveis já foram atualizadas desde então.

\section{Referências Bibliográficas}

ALÁSTICO, Gabriel Pedro; TOLEDO, José Carlos De. Acreditação Hospitalar: proposição de roteiro para implantação. Gestão \& Produção, São Carlos, v. 20, n. 4, p. 815-831, 2013.

BRASIL. Portaria 1101/GM, de 12 de junho de 2002. Estabelece os parâmetros de cobertura assistencial no âmbito do Sistema Único de Saúde - SUSBrasíliaMinistério da Saúde, , 2002. Disponível em: <http://www1.saude.ba.gov.br/regulasaude/2009/PN PORTARIAS 2009/nvos pdfs 2009/PT GM 1101 12.06.2002.pdf>

GÓES, Ronald De. Manual prático de arquitetura hospitalar. 2. ed. São Paulo: Blucher, 2011.

GUELLI, A.; ZUCCHI, P. A influência do espaço físico na recuperação dos pacientes e os sistemas e instrumentos de avaliação. Revista de Administração em Saúde, São Paulo, v. 7, n. 27, abr-jun, p. 43-50, 2005.

GUELLI, Augusto. Sistema de avaliação de edifícios de saúde. Pós: Revista do Programa de Pós-Graduação em Arquitetura e Urbanismo da FAUUSP, [s. I.], v. 0, n. 27, p. 174-192, 2010.

HELENE, Paulo R. L. Manual para reparo, reforço e proteção de estruturas de concreto. 2. ed. São Paulo: PINI, 1992.

HOLANDA, Frederico De. Brasília - Cidade moderna, cidade eterna. Brasília: FAU/Unb, 2010.

KARMAN, Jarbas. Manutenção hospitalar preditiva. 1. ed. São Paulo: PINI, 1994.

KOHLSDORF, Gunter; KOHLSDORF, Maria Elaine. Ensaio sobre o desempenho morfológico dos lugares. Brasília: FRBH, 2017.

LIMA, João Filgueiras. Arquitetura: uma experiência na área da saúde. 1. ed. São Paulo: Romano Guerra Editora, 2012.

MASCARÓ, Juan Luis. O custo das decisões arquitetônicas. São Paulo: Nobel, 1985.

OLIVEIRA, Janes Cleiton Alves De. Custos na construção civil voltados à área de saúde. In: Tecnologia e sustentabilidade para a humanização dos edifícios de saúde. 2. ed. Brasíla: FAU/Unb, 2016. p. 381-418.

ONO, Rosária et al. Avaliação de sistemas construtivos em habitações econômicas em contextos inovadores no Brasil: a contribuição da avaliação pós-ocupaçãoCongresso Internacional Habitação no Espaço Lusófono (CIHEL)Laboratório Nacional de Engenharia Civil-LNEC, , 2013.

ONO, Rosária et al. Avaliação de desempenho em uso e manutenção de habitações em sistemas construtívos inovadores. In: Avaliação de Desempenho de Tecnologias Construtivas Inovadoras: Conforto Ambiental, Durabilidade e Pós-Ocupação. [s.I.] : ANTAC, 2017.

ORNSTEIN, Sheila. Avaliação pós-ocupação (APO) do ambiente construído. São Paulo: Nobel/Fauusp, 1992.

ORNSTEIN, Sheila Walbe. Periferia Paulistana da necessidade de inserção das habitações de interesse social na Malha Urbana. Pós: Revista do Programa de Pós-Graduação em Arquitetura e Urbanismo da FAUUSP, [s. I.], v. 1, n. 1, p. 39-48, 1990.

ORNSTEIN, Sheila Walbe. Postoccupancy Evaluation Performed in Elementary and High Schools of Greater São Paulo, Brazil. Environment and Behavior, [s. I.], v. 29, n. 2, p. 236-263, 1997. Disponível em: <http://journals.sagepub.com/doi/10.1177/001391659702900205>

ORNSTEIN, Sheila Walbe et al. Performance evaluation of a psychiatric facility in São Paulo, Brasil. Facilities, Bradford, v. 27, n. 3/4, p. 152-167, 2009.

ORNSTEIN, Sheila Walbe. Avaliação Pós-Ocupação (Apo) No Brasil, 30 Anos: O Que Há De Novo? Revista Projetar - Projeto e Percepção do Ambiente, [s. I.], v. 2, n. 2, p. 7-12, 2017. Disponível em: <http://www.revistaprojetar.ct.ufrn.br/index.php/revprojetar/article/view/209/11>

ORNSTEIN, Sheila Walbe; DEL CARIO, Ualfrido. Tecnologia da arquitetura e a pós na FAUUSP: um quarto de século atuando no desenvolvimento das pesquisas em avaliação do desempenho ambiental. Pós. Revista do Programa de Pós-Graduação em Arquitetura e Urbanismo da FAUUSP, [s. I.], n. 8, p. 116, 2017.

Avaliação de Desempenho por meio de Ensaio Morfológico aplicado a arquitetura hospitalar 
ORNSTEIN, Sheila Walbe; LEITE, Brenda Chaves Coelho; ANDRADE, Cláudia Miranda. Office spaces in Sao Paulo: post-occupancy evaluation of a high technology building. Facilities, Bradford, v. 17, n. 11, p. 410, 1999.

ORNSTEIN, Sheila Walbe; ONO, Rosaria Rosária; OLIVEIRA, Fabiana Lopes De. Em busca da qualidade na habitação social no Brasil: instrumentos para a Avaliação Pós-Ocupação (APO) aplicada a sistemas construtivos inovadores. In: CONGRESSO INTERNACIONAL DA HABITAÇÃO NO ESPAÇO LUSÓFONO (CIHEL), 4., 2017, Covilha. Anais... Covilha: Universidade da Beira Interior, 2017.

ROMÉRO, Marcelo de Andrade. Retrofit e APO - Conforto Ambiental e Conservação de Energia/Eficiência Energética. In: Tecnologia e sustentabilidade para a humanização dos edifícios de saúde. 2. ed. Brasíla: FAU/Unb, 2016. p. 12-57.

ROMERO, Marta Adriana Bustos; FERNANDES, Júlia Teixeira. Sustentabilidade dos edifícios de saúde. In: Tecnologia e sustentabilidade para a humanização dos edifícios de saúde. 2. ed. Brasília: FAU/Unb, 2016. p. 60-139.

ROMERO, Marta Adriana Bustos; SALES, Gustavo de Luna (org. .. Tecnologia e sustentabilidade para a humanização dos edifícios de saúde: registro do curso de capacitação em arquitetura e engenharia aplicado a área de saúde, hemoterapia e hematologia. 2. ed. Brasília: FAU/UNB, 2016.

ROMERO, Marta Adriana Bustos; TEIXEIRA, Ederson Oliveira. Hemorrede Sustentável: A reabilitação ambiental sustentável dos edifícios dos Hemocentros Coordenadores. A política da Humanização do SUS. In: Tecnologia e sustentabilidade para a humanização dos edifícios de saúde. 2. ed. Brasíla: FAU/Unb, 2016. p. 420-448.

SOUZA, Vicente Custódio Moreira De; RIPPER, Thomaz. Patologia, recuperação e reforço de estruturas de concreto. São Paulo: PINI, 1998.

TENÓRIO, Gabriela de Souza. Ao Desocupado em Cima da Ponte: Brasília, arquitetura e vida pública. 2012. Tese (Doutorado em Arquitetura e Urbanismo) Dissertação (Mestrado em Arquitetura e Urbanismo) Faculdade de Arquitetura e Urbanismo, Universidade de Brasília, Brasília, 2012. Disponível em: <http://repositorio.unb.br/bitstream/10482/10710/1/2012_GabrieladeSouzaTenorio.pdf>

THOMAZ, Ércio. Trincas em edificios : Causas, prevenção e recuperação. São Paulo: PINI, 1989. 